HAEMATOLOGICAL CANCER

\title{
Brentuximab vedotin - a new standard for cutaneous T-cell lymphoma
}

Cutaneous T-cell lymphomas, including mycosis fungoides (MF) and primary cutaneous anaplastic large-cell lymphoma (pcALCL), are generally incurable, and have considerable detrimental effects on quality of life and, often, survival. Treatment options are limited, particularly for advanced-stage disease, and no preferred systemic therapy has been established. "Chemotherapy is largely ineffective, with responses lasting only a few months, and standard therapies, such as bexarotene and methotrexate, are similarly inadequate," explains Miles Prince. These lymphomas typically express CD30; hence, Prince et al. have now compared the anti-CD30antibody-drug conjugate brentuximab vedotin with physician's choice of bexarotene or methotrexate in a phase III trial involving 131 patients with refractory MF or PcALCL. Their findings confirm the impressive results of previous phase II studies. "We demonstrated marked improvements in the objective response rate in the brentuximab arm (67\% versus $20 \%$ ), as well as the rate of objective responses lasting $\geq 4$ months ( $56 \%$ versus $25 \%$ ), and in particular progression-free survival (16.7 months by EMA criteria, or 17.2 months by the FDA criteria, versus only 3.5 months)." Of note, durable responses were seen across the observed range of lymphoma-cell CD30-positivity (3-100\%). "These findings will be practice-changing and brentuximab vedotin will become a key therapy for patients with MF or pcALCL," Prince opines.

The rate of grade 3-4 adverse events was similar between the treatment cohorts $(41 \%$ versus $47 \%$ ), although peripheral neuropathy was observed in $67 \%$ of the brentuximab cohort versus $6 \%$ of the physician's choice cohort. Thus, priorities for further studies include "ways to reduce the incidence of neuropathy, moving the drug forward into earlier disease settings, and combination therapies," Prince concludes.

David Killock

ORIGINAL ARTICLE Prince, H. M. et al. Brentuximab vedotin or physician's choice in CD30-positive cutaneous T-cell lymphoma (ALCANZA): an international, open-label, randomised, phase 3 , multicentre trial. Lancet http://dx.doi.org/10.1016/S0140-6736(17)31266-7 (2017) 